\title{
Editorial
}

\section{Advances of Human Factors Research for Future Vehicular Technology}

\author{
Motoyuki Akamatsu, ${ }^{1}$ Paul Green, ${ }^{2}$ and Klaus Bengler ${ }^{3}$ \\ ${ }^{1}$ Human Technology Research Institute, AIST, Japan \\ ${ }^{2}$ University of Michigan Transportation Research Institute (UMTRI), USA \\ ${ }^{3}$ Institute of Ergonomics, Technische Universität München, Germany \\ Correspondence should be addressed to Motoyuki Akamatsu; akamatsu-m@aist.go.jp
}

Received 20 August 2013; Accepted 20 August 2013

\begin{abstract}
Copyright (C 2013 Motoyuki Akamatsu et al. This is an open access article distributed under the Creative Commons Attribution License, which permits unrestricted use, distribution, and reproduction in any medium, provided the original work is properly cited.
\end{abstract}

Although automotive human factors research began after the World War II, vehicular technology has developed to adapt the vehicle to the human operator and the requirements of traffic since its inception, with the initial focus being on ease of operation of the steering wheel and brake pedal and methods to provide adequate road illumination at night. For many decades, human factors research mainly concerned making the primary driving tasks (controlling a vehicle and obeying signs and signals) easy to do, providing adequate space for the driver and passengers, mitigating crash injuries, and making secondary controls and displays inside the vehicle easy to use.

With the introduction of advanced driver assistance systems and driver information systems in 1980s, there have been a marked increase in the number of studies of driver mental workload as well as more general, quantitative studies of driver behavior, both on real roads and in driving simulators to help design and evaluate those systems. That line of human factors research will continue as vehicle automation and driver information increases. Another line of research concerns driver distraction, with a special concern being the use of mobile devices such as cellular phones. The implementation of driver assistance and information systems has resulted in a shift in automotive human factors research.

Methods for the automotive human factors research have been mostly transferred from psychology and cognitive science, physiology, statistics, and various engineering disciplines. But, as vehicular technology continues to evolve, new methods and theories are needed to address those issues and the human aspects of the vehicular technology so vehicles will be safe, easy to use, and useful.

The aim of the special issue is to collect research activities pertaining to human factors issue in the advanced driver assistance systems and driver information systems and to develop new methods for future automotive human factors issues.

The review article "Automotive technology and human factors research: past, present, and future" describes the history of automotive human factors research since the inception of motor vehicles until now. This article covers not only the research topics examined but also the industrial standards developed as a result of that research, the major organizations that research and conferences established at which it was presented, the major news stories affecting vehicle safety, and the general social context. It also identifies the issues to be addressed in the future.

We have two articles regarding the interface design of driver information systems. "Development and evaluation of automotive speech interfaces: useful information from the human factors and the related literature" by V. E. W. Lo, and P. A. Green provides a comprehensive literature review of the topic, summarizing much of what has been done in tables. Speech interfaces, though not commonly used now, have the potential of being less distracting to the driver than the visual-manual interfaces. The review article gives the background information such as key speech interfaces 
(both demonstrations and products), relevant technology standards and guidelines, and assessment methods for future development. "Evaluation of a navigation radio using the think-aloud method" by P. A. Green and J.-S. Park is a research article that describes how problems associated with a preproduction speech interface of a driver information system were identified using the think-aloud method commonly applied for usability testing. In addition to providing an extensive list of problems (common to many speech interfaces) as well as their frequency and severity, this article provides suggestions as to how the think-aloud method can be improved in this context so data can be collected and analyzed more quickly at a lower cost.

We have two research articles for assessing driver's capability of the driving task based on driving performance. "Analysis of temporal relationships between eye-gaze and peripheral vehicle behavior for detecting driver distraction" by $\mathrm{T}$. Hirayama et al. gives a new measure to assess the driver distraction. They focused on the gaze behavior towards the peripheral vehicles and see how it changes depending on the level of distraction. "A neurofuzzy approach to modeling longitudinal driving behavior and driving task complexity" by R. G. Hoogendoorn, et al. focuses on the performance of the longitudinal driving task and how it changes depending on the driving task complexity, based on the theoretical framework of Task-Capability-Interface model that goes well beyond the classical Gazis-Herman-Rothery and Intelligent Driver models in the literature. They developed a mathematical model that can be used to assess the driver capability.

Overtrust and overreliance are key concerns when introducing advanced driver assistance systems but they are not clearly understood. "Human's overtrust in and overreliance on advanced driver assistance systems: a theoretical framework" by $\mathrm{T}$. Inagaki and M. Itoh gives a two-aspect framework for the system (system diagnosis, action selection). System diagnosis is used to determine overtrust and has three characteristics (dimension of trust, target object, and chances for observation). The second aspect is used to determine overreliance and has three characteristics (type of action selected, benefits expected, and time allowance for intervention). Numerous examples of how these ideas can be applied to design real systems are given.

We have two research articles where the authors are developing methods for the human factors research. "Predicting driver behavior using field experiment data and driving simulator experiment data: assessing impact of elimination of stop regulation at railway crossings" by $\mathrm{T}$. Sato et al. showed how the combined use of a naturalistic driving experiment and a driving simulator experiment can predict how drivers brake and where they stop if stop signs are removed from railway crossings. Of particular concern was the situation where vehicles ahead stop and the driver is trapped in the crossing and possibly killed by an oncoming train. "The front seat passenger: how to transfer qualitative findings into design" by S. Osswald et al. describes how qualitative methods can be used to identify ideas for new vehicle design, methods that contrast with traditional, quantitative methods used to test hypotheses using statistics.
"A comparative analysis of subjective quality of the mobility between new portable electric transportation mode and walking" by $\mathrm{H}$. Ohta et al. is a research article concerning a Segway-type personal mobile vehicle. They investigated the subjective quality of mobility related to the use of this type of vehicle in different situations, to determine when it would be used and when people would prefer to walk or drive a car.

The variety of topics and methods described is indicative of the current state of the automotive human factors literature. For next generation motor vehicles and vehicle systems to be developed in a timely and cost-effective manner that customers will find useful, easy to use, and safe, efforts to (1) develop new methods, (2) collect and summarize the literature, (3) develop standards, guidelines, and recommended practices, and (4) evaluate prototype product designs must occur in parallel with human-centered design.

Motoyuki Akamatsu Paul Green Klaus Bengler 

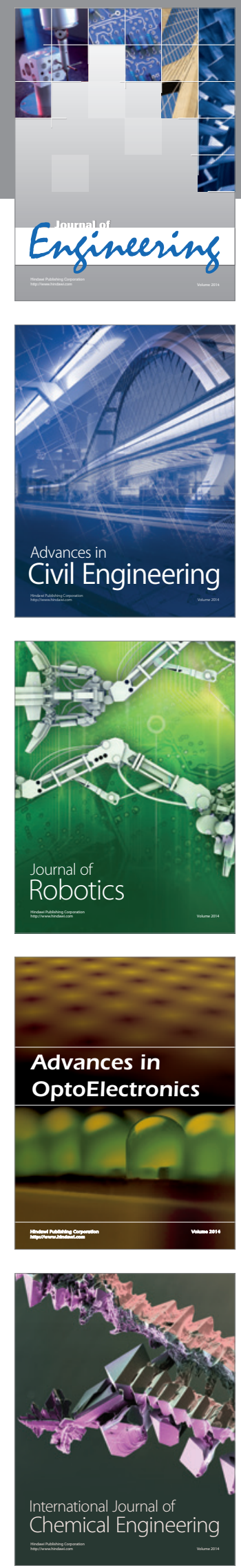

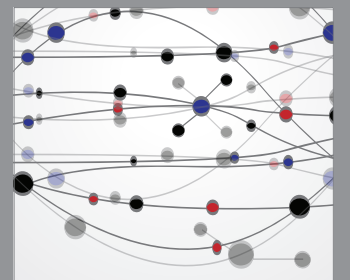

The Scientific World Journal
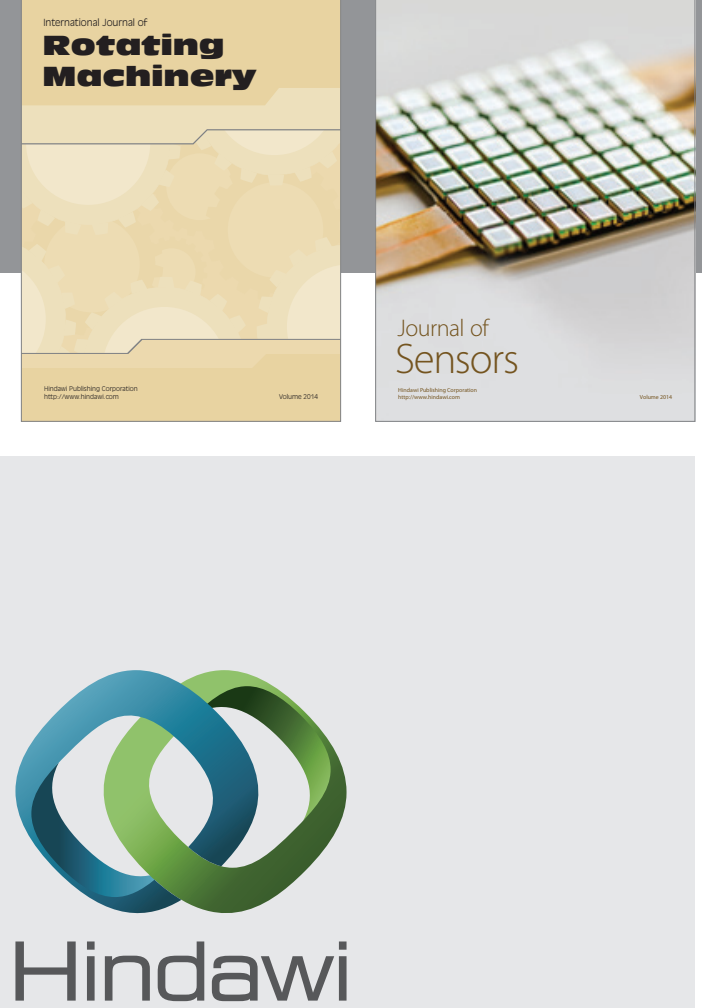

Submit your manuscripts at http://www.hindawi.com
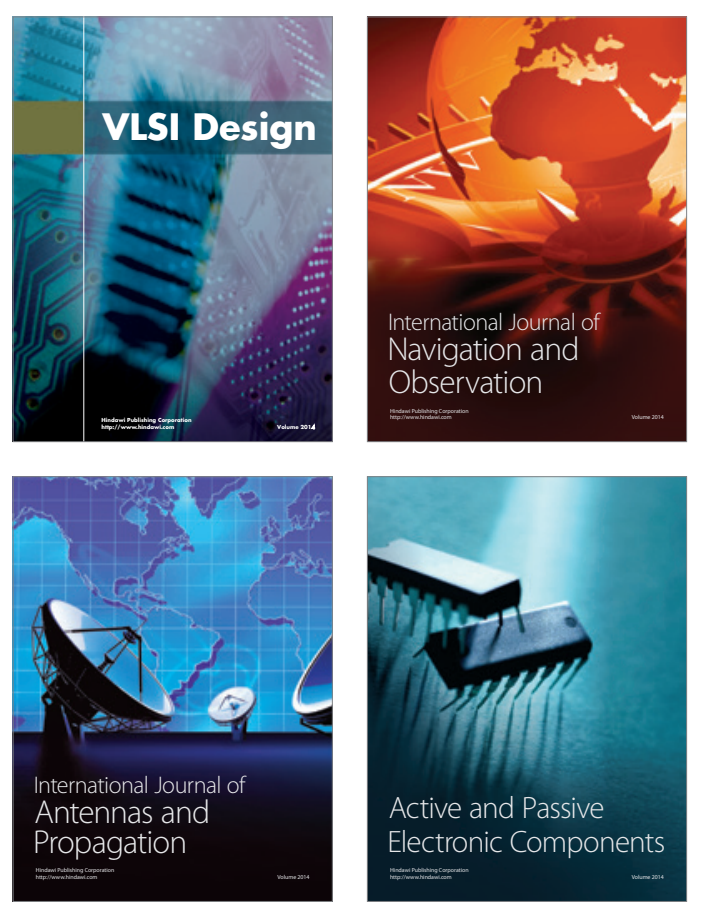
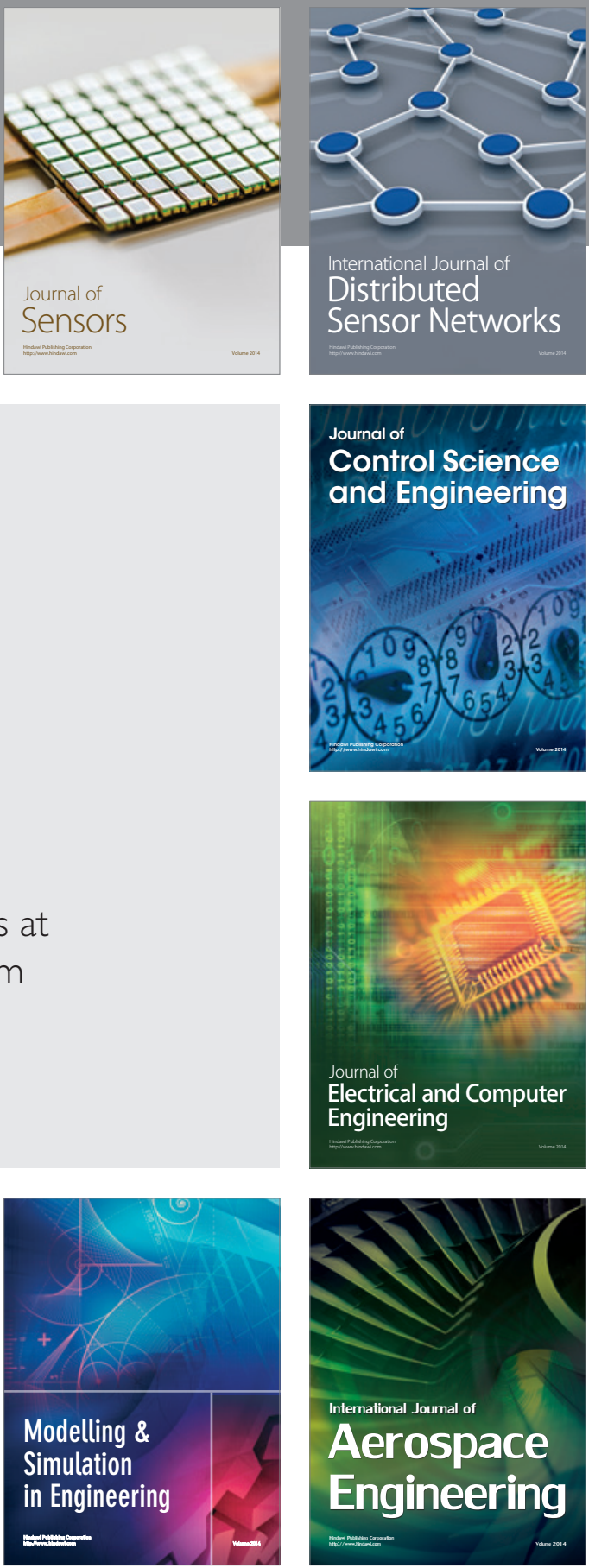

Journal of

Control Science

and Engineering
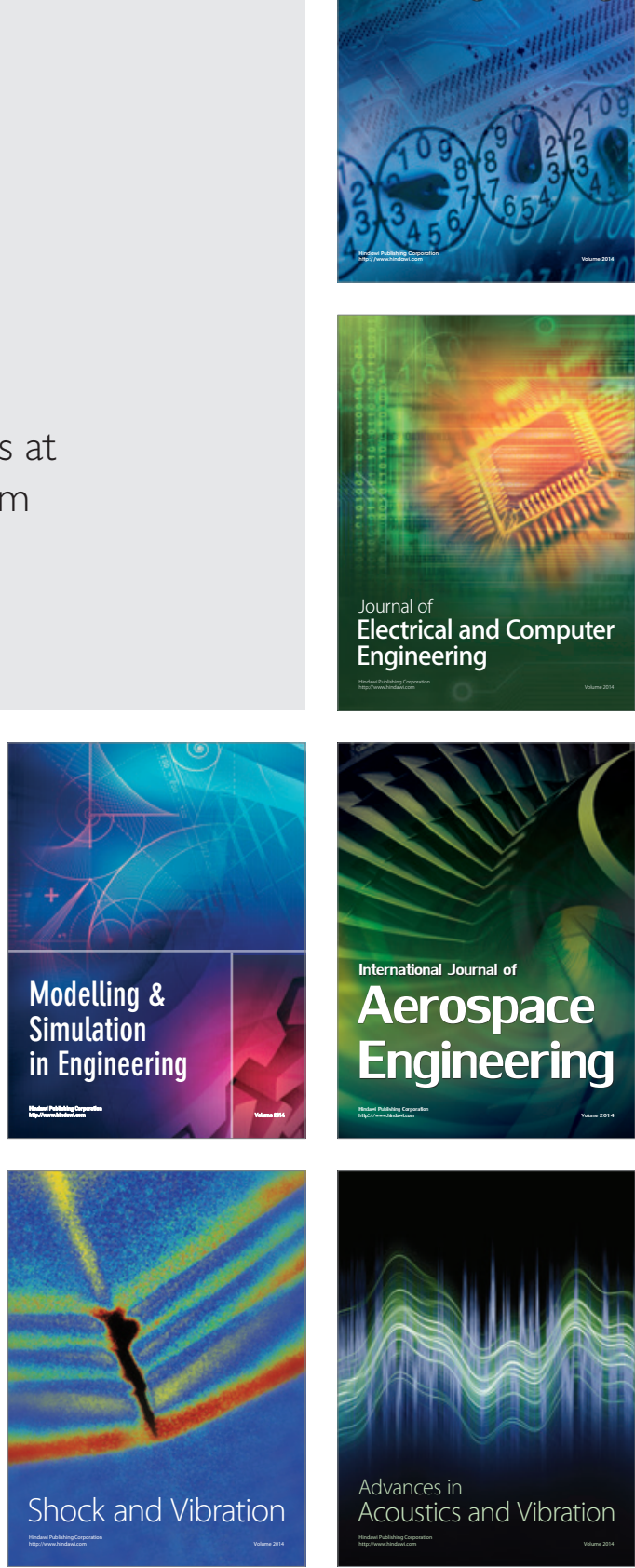